\title{
When the bond breaks - targeting adhesion of leukemia cells to the meninges
}

\section{Lennart Lenk, Fotini Vogiatzi and Denis M. Schewe}

Department of Pediatrics I, ALL-BFM Study Group, Christian-Albrechts University Kiel and University Medical Center SchleswigHolstein, Kiel, Germany.

E-mail: DENIS M. SCHEWE - Denis.Schewe@uksh.de

doi:10.3324/haematol.2020.253609

A major clinical challenge in the treatment of acute lymphoblastic leukemia (ALL) is the involvement of the central nervous system (CNS), especially in children. CNS involvement can occur upon initial diagnosis and be a source of relapse. ${ }^{1}$ Due to the high propensity of ALL cells to infiltrate the CNS and the lack of specific markers for their detection and eradication, patients receive high cumulative doses of intrathecal chemotherapy. This is associated with neurotoxicity, which may lead to acute complications and long-term developmental delay in children. ${ }^{2}$ The CNS is regarded as a privileged "niche" in which ALL cells can persist due, for example, to the paucity of immune cells and the blood-brain barrier impairing accessibility of this sanctuary by chemotherapy. ${ }^{3,4}$ Thus, gaining a deeper understanding of how ALL cells interact with the CNS microenvironment may open the field for novel targeted approaches to eradicate ALL cells in the CNS. In this issue of Haematologica, Jonart et al. describe how ALL cells seek shelter by adhering to meningeal cells, resulting in quiescence and chemoresistance. The authors propose interference with adhesion mechanisms as a novel therapeutic strategy in CNS leukemia ${ }^{5}$ (Figure 1).

Infiltration of the CNS can be observed in metastasis of solid cancers, as well as in hematologic malignancies including ALL, other leukemias and lymphomas. CNS metastasis of solid cancers mostly affects the brain parenchyma, but ALL cells predominantly reside in the leptomeninges, a conjunctive tissue surrounding the parenchyma and the ventricular choroid plexus. ${ }^{5-7}$ Hence, the mech-

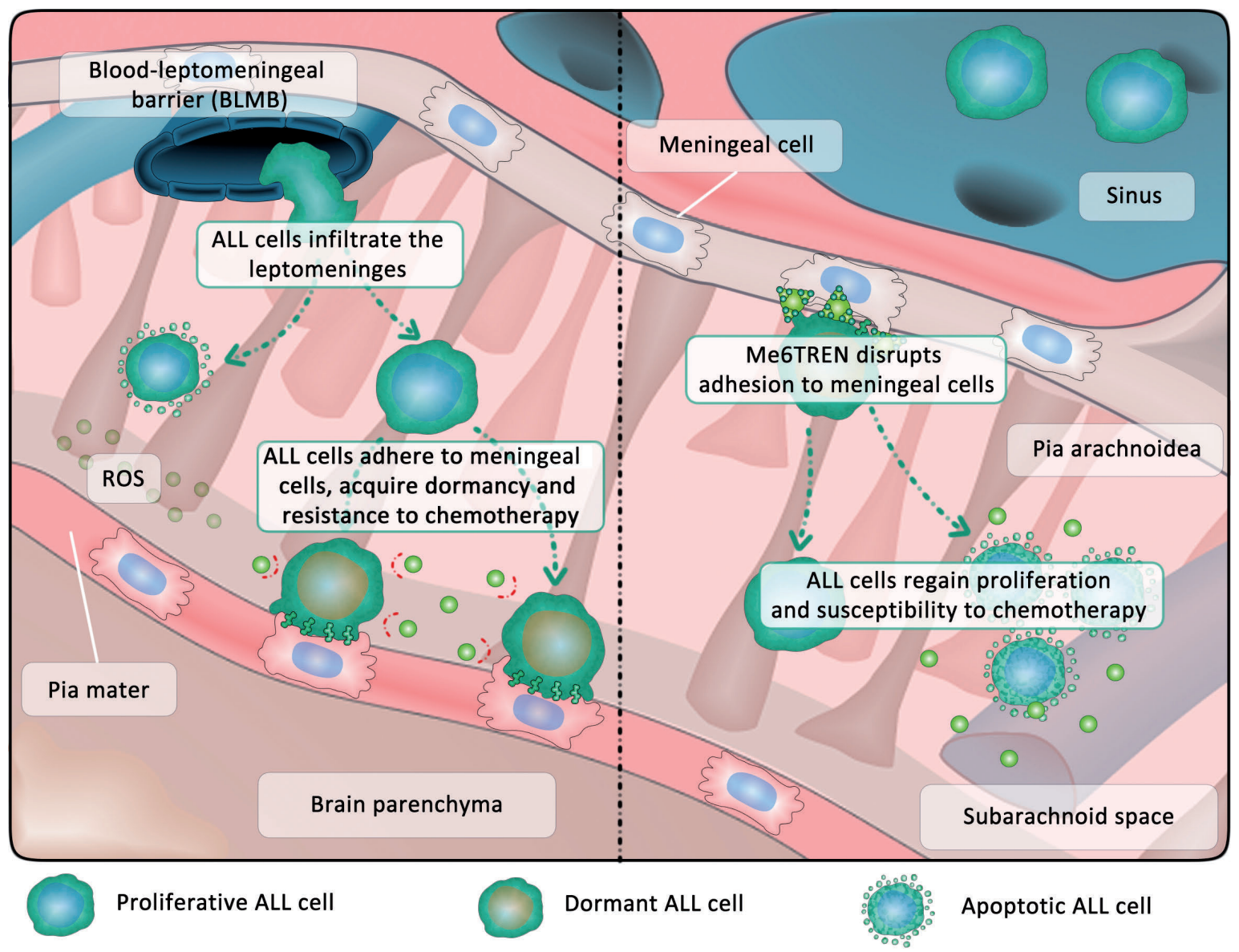

Figure 1. Leukemia cells seek shelter by adhering to meningeal cells. Acute lymphoblastic leukemia (ALL) cells breach the blood-leptomeningeal barrier and infiltrate the leptomeninges. They encounter a hostile microenvironment in which nutrients are scarce (cerebrospinal fluid) and levels of reactive oxygen species (ROS) are enhanced. By adhering to meningeal cells, ALL cells acquire a state of reversible quiescence (dormancy) rendering them less vulnerable to chemotherapy. Disrupting the adhesion of ALL cells to meningeal cells via Me6TREN mobilizes ALL cells from the niche, thereby restoring their susceptibility to chemotherapeutic agents. 
anisms of CNS infiltration in solid tumors and hematologic malignancies likely differ fundamentally. To enter the CNS environment, ALL cells need to pass protective physiological barriers such as the blood-brain barrier, the blood-leptomeningeal barrier and the blood-cerebrospinal fluid barrier, which in physiological conditions ensure the controlled flux of molecules and passage of cells into the organ. ${ }^{3}$ The cerebrospinal fluid per se may also represent a hostile environment for ALL cells due to the limited quantity of proteins and the presence of reactive oxygen species. ${ }^{8}$ The CNS microenvironment may, therefore, critically affect the behavior of ALL cells in this niche.

To investigate the behavior of ALL cells in the CNS, Jonart et al. co-cultured B-cell precursor ALL and T-ALL cell lines as well as primary B-cell precursor ALL cells with primary human meningeal cells, modeling the leptomeningeal microenvironment. ${ }^{5}$ The authors found that ALL cells efficiently adhered to the primary human meningeal cells, rendering them less vulnerable to chemotherapy. Therapy sensitivity was markedly reduced when ALL cells were grown in direct contact with the meningeal cells and treated with methotrexate and cytarabine, two major compounds of CNS-directed chemotherapy in ALL. Direct contact with primary human meningeal cells also reduced proliferation of ALL cells, leading to the conclusion that chemoresistance was based on the acquisition of a dormant phenotype. Cellular dormancy is a state of transient $G_{0} / G_{1}$ arrest that may be induced by hostile microenvironments and that may be reversed when cells encounter favorable conditions again. ${ }^{9}$ Evidence that dormancy may represent a key mechanism of CNS disease and relapse in ALL is accumulating beyond this work. ${ }^{3}$ For example, patient-derived xenograft ALL cells recollected from murine meninges were shown to be less proliferative than cells recollected from the bone marrow..$^{10}$ Furthermore, direct co-culture models of CNS leukemia showed $\mathrm{G}_{0} / \mathrm{G}_{1}$ arrest and resistance to methotrexate-mediated cytotoxicity in vitro in B-cell precursor ALL in dependence of the receptor tyrosine kinase MER. ${ }^{11}$ Adhesion processes of leukemia cells to CNS microenvironmental structures have also recently been shown to play a crucial role in the invasion of leukemia cells into the CNS. Yao et al. showed that the interaction of leukemic cells expressing $\alpha 6$-integrin with laminin on the abluminal surface of emissary blood vessels facilitates the cells' entry into the CNS. ${ }^{12}$ Furthermore, the chemokine receptor CCR7 was found to promote adhesion and trafficking across the choroid plexus in T-ALL. ${ }^{13}$ With their recent report, Jonart et al. provide a further piece of the puzzle of CNS leukemia as they show that meningeal cells, presumably the cell entity that ALL cells mostly encounter in the CNS microenvironment after invading through the vasculature, promote ALL dormancy, survival and therapy resistance. This observation has important implications for understanding the mechanistic basis of CNS infiltration and relapse in ALL.

The study by Jonart et al. raises the questions of whether the acquisition of dormancy by adhesion to meningeal cells is a feature of every ALL cell in the CNS or whether this is only relevant in a fraction of cells undergoing selection by chemotherapy. Or, if all leukemic cells reaching the CNS adhere to meningeal cells and cease proliferation, how would they then go on to colonize the meninges and be clinically detectable? In their study, Jonart et al. detected a small, slow cycling fraction of ALL cells recovered from the meninges of xenografted mice. Intriguingly, after treating leukemia-bearing mice with cytarabine, the authors found a 13-fold increase in the number of these quiescent cells whereas only a 5 -fold relative increase was found in the bone marrow. This argues in favor of a small fraction of dormant cells that may survive treatment, as previously suggested, ${ }^{14}$ and in favor of the CNS microenvironment fostering the maintenance and enrichment of this fraction. Various molecules and pathways have recently been identified to be associated with CNS infiltration and relapse, some of which are also linked to cellular adhesion: the ZAP70 kinase regulates CXCR 4 and CCR $7,{ }^{15}$ the receptor tyrosine kinase MER exposes structural similarity to neural adhesion molecules ${ }^{11,16}$ and phosphoinositide-3-kinase was shown to promote integrin-mediated adhesion. ${ }^{12}$ It would be interesting to investigate whether cells enriching in the CNS after chemotherapy show a particularly high expression of these molecules.

If adhesion-mediated chemoresistance contributes to CNS relapse, how can this concept be exploited to establish novel targeted therapy approaches? One substantial advantage of compounds targeting general features such as adhesive interactions could be that they act on a variety of ALL cells irrespective of the genetic background. A previous report described promising results obtained from disrupting $\alpha 6$ integrin-laminin interactions via inhibition of phosphoinositide-3-kinase which delayed leukemic CNS engraftment in xenograft mouse models. However, the inhibitors used in this study, including idelalisib, which is already in clinical use, were shown to be unable to cross the bloodbrain barrier. ${ }^{12}$ Therefore, such approaches may be effective in preventing the entry of ALL cells into the CNS rather than targeting ALL cells already residing in the CNS. ${ }^{12}$ CXCR4 is another key molecule associated with homing and adhesion processes in ALL in the CNS..$^{15}$ The CXCR4 antagonist AMD3100 (plerixafor) reduced leukemia burden in peripheral organs of xenograft mice, but not in the CNS. ${ }^{\circ}$ Testing different compounds, Jonart et al. found a high efficacy of the small molecule inhibitor Tris[2-(dimethylamino)ethyl]amine) (Me6TREN, Me6) in preventing the adhesion of ALL cells to meningeal cells in vitro, thereby restoring their sensitivity to cytarabine. When applied to mice bearing ALL cells, the addition of Me6TREN to cytarabine treatment resulted in diminished leukemic engraftment in the meninges as compared to that following treatment with cytarabine alone. Me6TREN was first described as a compound mobilizing hematopoietic progenitor cells from the bone marrow by upregulating matrix metalloprotease-9 and disrupting the CCL12/CXCR4 axis, thereby outperforming AMD3100. ${ }^{17}$ Accordingly, compared to AMD3100, Me6TREN showed enhanced efficacy in disturbing the adhesion of ALL cells to meningeal cells in the work by Jonart et al. ${ }^{5}$ Me6TREN has proven to be well tolerated in mice. ${ }^{17}$ If Me6TREN is demonstrated to penetrate the CNS in pharmacokinetic and pharmacodynamics studies, this compound could indeed be considered as a drug the for prevention and therapy of CNS disease.

Overall the study by Jonart et al. provokes some interesting questions for the future: Do the different cell types in the meninges, e.g., fibroblasts, endothelial cells and pericytes, have different impacts on CNS-infiltrating ALL cells? 
Are meningeal cells also involved in specific homing of ALL cells into the CNS? Is adhesion-mediated chemoresistance of ALL cells in contact with meningeal cells simply a cause of decreased proliferation and therefore diminished vulnerability to chemotherapeutic agents or is an active process involved (e.g., regulation of drug transporters)? Furthermore, it would be interesting to determine whether adhesion capacity of ALL cells to the CNS microenvironment could be used to improve CNS diagnostics. A recent report suggests that surface expression of $\alpha 5$-integrins on ALL cells is associated with the number of ALL cells in the cerebrospinal fluid detectable by diagnostic lumbar puncture. ${ }^{18}$ Finally, there is a need to consider that mobilizing dormant ALL cells by breaking adhesive bonds with meningeal cells may also confer potential risks. Re-awakening leukemic cells may cause a resumption of proliferation and therefore overt CNS disease, an aspect which will have to be clarified in the future.

The recent study by Jonart et al. shapes a sharper image of the complex mechanisms of both CNS infiltration and CNS relapse, and may ultimately contribute to improved strategies for targeted treatment of CNS leukemia in ALL.

\section{References}

1. Pui CH, Howard SC. Current management and challenges of malignant disease in the CNS in paediatric leukaemia. Lancet Oncol. 2008;9(3):257-268

2. Cheung YT, Khan RB, Liu W, et al. Association of cerebrospinal fluid biomarkers of central nervous system injury with neurocognitive and brain imaging outcomes in children receiving chemotherapy for acute lymphoblastic leukemia. JAMA Oncol. 2018;4(7):e180089.

3. Lenk L, Alsadeq A, Schewe DM. Involvement of the central nervous system in acute lymphoblastic leukemia. Opinions on molecular mechanisms and clinical implications based on recent data. Cancer Metastasis Rev. 2020;39(1):173-187

4. Frishman-Levy L, Shemesh A, Bar-Sinai A. et al. Central nervous sys- tem acute lymphoblastic leukemia: role of natural killer cells. Blood. 2015;125(22):3420-3431.

5. Jonart LM, Ebadi M, Basile P, et al. Disrupting the leukemia niche in the central nervous system attenuates leukemia chemoresistance. Haematologica. 2020;105(8):2130-2140.

6. Williams MTS, Yousafzai YM, Elder A, et al. The ability to cross the blood-cerebrospinal fluid barrier is a generic property of acute lymphoblastic leukemia blasts. Blood. 2016;127(16):1998-2006.

7. Price RA. Histopathology of CNS leukemia and complications of therapy. Am J Pediatr Hematol Oncol. 1979;1(1):21-30.

8. Basile P, Jonart LM, Ebadi M, Johnson K, Kerfeld M, Gordon PM. The meninges enhance leukaemia survival in cerebral spinal fluid. Br J Haematol. 2020;189(3):513-517.

9. Aguirre-Ghiso JA. Models, mechanisms and clinical evidence for cancer dormancy. Nat Rev Cancer. 2007;7(11):834-846.

10. Kato I, Nishinaka Y, Nakamura M, et al. Hypoxic adaptation of leukemic cells infiltrating the CNS affords a therapeutic strategy targeting VEGFA. Blood. 2017;129(23):3126-3129.

11. Krause S, Pfeiffer C, Strube S, et al. Mer tyrosine kinase promotes the survival of $t(1 ; 19)$-positive acute lymphoblastic leukemia (ALL) in the central nervous system (CNS). Blood. 2015;125(5):820-830.

12. Yao H, Price TT, Canelli G, et al. Leukaemia hijacks a neural mechanism to invade the central nervous system. Nature. 2018:560(7716):55-60

13. Buonamici S, Trimarchi T, Ruocco MG, et al. CCR7 signalling as an essential regulator of CNS infiltration in T-cell leukaemia. Nature. 2009:459(7249):1000-1004

14. Ebinger S, Özdemir EZ, Ziegenhain C, et al. Characterization of rare, dormant, and therapy-resistant cells in acute lymphoblastic leukemia. Cancer Cell. 2016;30(6):849-862 .

15. Alsadeq A, Fedders H, Vokuhl C, et al. The role of ZAP70 kinase in acute lymphoblastic leukemia infiltration into the central nervous system. Haematologica. 2017;102(2):346-355.

16. Chen J, Carey K, Godowski PJ. Identification of Gas6 as a ligand for Mer, a neural cell adhesion molecule related receptor tyrosine kinase implicated in cellular transformation. Oncogene. 1997;14(17):20332039

17. Zhang J, Ren X, Shi W, et al. Small molecule Me6TREN mobilizes hematopoietic stem/progenitor cells by activating MMP-9 expression and disrupting SDF-1/CXCR4 axis. Blood. 2014;123(3):428-441

18. Shah Scharff BFS, Modvig S, Thastrup M, et al. A comprehensive clinical study of integrins in acute lymphoblastic leukemia indicates a role of $\alpha 6 / C D 49 \mathrm{f}$ in persistent minimal residual disease and $\alpha 5$ in the colonization of cerebrospinal fluid. Leuk Lymphoma. $2020 \mathrm{Feb}$ 28;1-5. [Epub ahead of print]

\title{
BCL2 dependency in diffuse large B-cell lymphoma: it's a family affair
}

\author{
Shannon M. Matulis and Lawrence H. Boise
}

Department of Hematology and Medical Oncology Emory School of Medicine and the Winship Cancer Institute, Emory University; Atlanta, GA, USA

E-mail: LAWRENCE H. BOISE - lboise@emory.edu

doi:10.3324/haematol.2020.253591

$\mathrm{D}$ iffuse large B-cell lymphoma (DLBCL) is the most common form of non-Hodgkin lymphoma, accounting for approximately $25 \%$ of all lymphomas. ${ }^{1}$ DLBCL is highly heterogeneous, so responses to standard therapy, R-CHOP (rituximab plus cyclophosphamide, doxorubicin, vincristine, and prednisone) are mixed. ${ }^{2}$ The response, as well as mechanisms of resistance to therapy, are associated with a cell's apoptotic threshold. ${ }^{3}$ Therefore, determining the molecular basis for a tumor's ability to survive can provide insights into drug resistance as well as opportunities for precision medicine. In this issue of Haematologica, Smith et al. demonstrate the importance of the BCL2 family of anti-apoptotic proteins BCL2, BCLXL, and MCL1 in the survival of DLBCL, potentially revealing new treatment strategies. ${ }^{4}$

Inappropriate activation of oncogenes can result in cell death through the activation of pro-apoptotic proteins of the BCL2 family. Therefore, to survive the transformation process, tumor cells become more dependent on their anti-apoptotic BCL2 proteins (e.g., BCL2, BCLXL, and MCL1) than their normal counterparts. ${ }^{5.7}$ This dependency is the result of binding and neutralizing the pro-apoptotic family members (e.g., BIM, BAK, and BAX) and is often referred to as mitochondrial priming, as increased 